\title{
Insomnia is Associated with the Effect of Inpatient Multimodal Occupational Rehabilitation on Work Participation in Workers with Musculoskeletal or Mental Health Disorders: Secondary Analyses of a Randomized Clinical Trial
}

Eivind Schjelderup Skarpsno (iD 1,2 Sigmund Østgård Gismervik ${ }^{1,3}$ Marius Steiro Fimland ${ }^{4,5}$

Lene Aasdahl (iD) 1,5

'Department of Public Health and Nursing, Faculty of Medicine and Health Sciences, Norwegian University of Science and Technology (NTNU), Trondheim, Norway; ${ }^{2}$ Department of Neurology and Clinical Neurophysiology, St. Olavs Hospital, Trondheim, Norway; ${ }^{3}$ Department of Physical Medicine and Rehabilitation, St. Olavs Hospital, Trondheim University Hospital, Trondheim, Norway; ${ }^{4}$ Department of Neuromedicine and Movement Science, Faculty of Medicine and Health Sciences, NTNU, Norwegian University of Science and Technology, Trondheim, Norway;

${ }^{5}$ Unicare Helsefort Rehabilitation Centre, Rissa, Norway
Correspondence: Eivind Schjelderup Skarpsno

Department of Public Health and Nursing, Norwegian University of Science and Technology (NTNU), Trondheim, 749I, Norway

Tel +47 9752/297

Email eivind.s.skarpsno@ntnu.no
Study Objectives: Insomnia is common among people with musculoskeletal and/or mental health disorders. This study aimed to assess whether insomnia is associated with the favorable effect from inpatient multimodal occupational rehabilitation on future work participation among individuals with these conditions.

Methods: Insomnia was measured at baseline through a randomized clinical trial that compared the effect of inpatient multimodal occupational rehabilitation with a lesscomprehensive program of outpatient acceptance and commitment therapy on future work participation. The inpatient multimodal program lasted 3.5 weeks at the rehabilitation center, comprising psychoeducational sessions (including sleep education), fixed schedules, acceptance and commitment therapy, physical exercise and work-related problem-solving, whereas the outpatient program comprised mainly six weekly acceptance and commitment therapy sessions. Both programs were group-based. The study tracked cumulative sick leave during the 12 months of follow-up using national registry data.

Results: Among the 163 adults included in this subgroup analysis, 56\% $(n=91)$ reported insomnia. Overall, we found statistical evidence of interaction between the occupational program and insomnia concerning cumulative sick leave $(\mathrm{p}=0.03)$. Compared with people without insomnia in the comprehensive inpatient multimodal program, people with insomnia had 12 (95\% CI: -48 to 24$)$ fewer days with sick leave if they participated in the inpatient program and 46 (95\% CI: 8 to 83$)$ more days if they participated in the outpatient program. Conclusion: These findings suggest that insomnia should be addressed specifically before individuals on sick leave are considered for participation in occupational rehabilitation and that individuals with insomnia may benefit in particular from inpatient rehabilitation.

Keywords: sleep problems, occupational therapy, work, chronic pain, mental health, sick leave

\section{Introduction}

Musculoskeletal and common mental health disorders are the greatest contributors to work disability in the Western world. ${ }^{1}$ To improve work functioning and reduce costs associated with work disability from these conditions, implementation of multimodal occupational programs for sick-listed workers is recommended. ${ }^{2}$ 
However, results on the effect of such programs are inconsistent, ${ }^{3-6}$ possibly because of the large heterogeneity among workers with musculoskeletal and mental health disorders. ${ }^{7,8}$ Considering the high costs of providing inpatient programs, it is essential to gain further insight about subgroups that would benefit the most.

Several studies suggest a strong link between insomnia and the risk of sick leave and work disability due to musculoskeletal and mental health disorders, ${ }^{9-11}$ and it has been implied that insomnia should be recognized and targeted in occupational rehabilitation and prevention programs. ${ }^{12}$ Insomnia is common among people with musculoskeletal disorders ${ }^{13}$ or mental health disorders, ${ }^{14}$ and it plays an important role in the risk and progression of these conditions. $^{15-20}$ Additionally, these conditions appear to be bidirectionally related, where musculoskeletal pain and mental health disorders also increase the risk of insomnia. $^{21,22}$ Inpatient rehabilitation programs incorporate principles known to be helpful for good sleep quality, ${ }^{23,24}$ such as physical exercise, ${ }^{25,26}$ psychoeducational sessions, including sleep education, and fixed schedules. ${ }^{27}$ However, few studies have examined whether insomnia is associated with the beneficial effect of such programs on future work participation. This knowledge may help determine who will benefit from such programs and provide valuable input on the development of effective interventions.

In a recent randomized clinical trial of sick-listed individuals with musculoskeletal or mental health disorders, we found that individuals participating in a 3.5-week-long inpatient multimodal occupational rehabilitation program had 32 fewer sickness absence days during 12 months of follow-up than a less-comprehensive outpatient program. ${ }^{24}$ The inpatient program consisted of various treatment modalities, such as physical exercise, psychoeducation, acceptance and commitment therapy, work-related problem solving and the development of a written return to work plan, while the outpatient program consisted mainly of acceptance and commitment therapy. ${ }^{24}$ The present study aimed to examine whether the favorable effect of the multimodal inpatient program on future work participation was associated with insomnia.

\section{Materials and Methods Study Design}

The study used a subgroup analysis of a randomized, controlled trial with 12 months of follow-up that aimed to evaluate the effect of inpatient, multimodal, occupational rehabilitation on future work participation. In the subgroup analyses, it was assessed whether the effect of the inpatient and outpatient programs on accumulated sick leave was different for participants with or without insomnia. More in-depth information about the trial can be found elsewhere. $^{24,28}$ The primary outcome of this trial is available in another publication. ${ }^{24}$

The Regional Committee for Medical and Health Research Ethics in Central Norway (No.: 2012/1241) approved the study.

\section{Participants}

Eligibility criteria for the trial were as follows: a) ages 1860 ; b) on sick leave for 2-12 months (with a current sick leave status of at least 50\%); and c) diagnosed within the musculoskeletal (L), psychological (P) or general and unspecified (A) chapters of the International Classification of Primary Care (second edition, ICPC-2). The exclusion criteria were as follows: a) alcohol or drug abuse; b) having a serious somatic (eg, cancer and unstable heart disease), psychiatric (eg, high suicidal risk, psychosis and ongoing manic episodes) or neurological (eg, multiple sclerosis, chronic fatigue syndrome/myalgic encephalomyelitis) disorder; c) having a specific disorder that requires specialized treatment; d) pregnancy; e) currently participating in another treatment or rehabilitation program; f) insufficient oral or written Norwegian language skills needed to participate in group sessions and fill out questionnaires; g) scheduled for surgery within the next six months; and h) serious problems with social functioning in a group setting.

\section{Interventions}

The inpatient multimodal occupational rehabilitation program was both individual and group-based (maximum of eight participants in each group) and lasted three-and -a-half weeks, for six to seven hours each day except on weekends. The program took place at the Hysnes Rehabilitation Centre, which was established as part of St. Olavs Hospital in central Norway, with two to three designated coordinators (eg, physical therapy, psychology, exercise physiology, nursing) per group involved in coordinating and executing the interventions. The coordinators were trained in return-to-work problem-solving. The program comprised three main areas of rehabilitation: "mental training"; "physical training"; and "work-related problem solving". ${ }^{28}$ The mental training comprised acceptance and 
commitment therapy, ${ }^{29}$ a form of cognitive-behavioral therapy, along with mindfulness and psychoeducation (sleep hygiene, stress, nutrition and pain). The physical training comprised group and individual-based exercises that aimed to increase physical strength and endurance capacity. The work-related problem-solving aimed to motivate the participant, clarify the value of work, highlight challenges and resources, and create a realistic return-to-work plan.

The comparative program comprised mainly of outpatient, group-based acceptance and commitment therapy. The participants were offered two-and-a-half-hour-long group sessions (with a maximum of nine participants in each group) once a week for six weeks. In addition to the acceptance and commitment therapy group sessions, the participants participated in one-group session with psychoeducation on physical activity, two individual sessions with a social worker and a short individual closing session with a group therapist (psychologist or physician) specifically trained in acceptance and commitment therapy. The sessions were held at the Department of Physical Medicine and Rehabilitation, St. Olavs Hospital. The participants were encouraged not to start any new treatment during the intervention; however, they could continue any treatment agreed upon by their general practitioner. Among the group sessions, participants were also encouraged to practice at home, including 15 minutes of daily mindfulness. A more detailed description of the programs has been published elsewhere. ${ }^{28}$

\section{Insomnia}

Insomnia was measured at baseline (before randomization) using the Insomnia Severity Index (ISI) ${ }^{30}$ which is viewed as a reliable and valid instrument for detecting insomnia cases within a population. ${ }^{31}$ The ISI comprises a sevenitem self-report questionnaire that assesses insomnia's nature, severity, and impact over the previous two weeks. The first three items assess the perceived severity of difficulties in initiating and maintaining sleep. The next four items assess satisfaction with current sleep patterns, interference with daily functioning, impairment due to sleep problems and distress/concerns regarding sleep problems. A 5-point Likert scale was used to rate each item $(0=$ no problems; 4 $=$ severe problems), providing for a maximum score of 28 , with high scores indicating greater insomnia severity. Considering that a cutoff score of $\geq 15$ may be too stringent for diagnosing patients and produce too many false negatives, ${ }^{31}$ the participants were dichotomized into "no insomnia" $(<11)$ and "insomnia" $(\geq 11)$. A cutoff $\geq 11$ was viewed as indicating high accuracy for diagnosing insomnia in a clinical sample. ${ }^{31}$

\section{Other Variables}

Information on demographic factors, such as gender, age (continuous variable) and education (categorized as high [college/university] or low), was obtained through questionnaires upon inclusion. Anxiety and depression symptoms were measured using the Hospital Anxiety and Depression scale (HADS), ${ }^{32}$ comprising 14 items in which seven measure anxiety symptoms and seven measure depression symptoms, all scored on a 4-point Likert scale according to intensity of symptoms last week, with a maximum score of 21 on each subscale. Average pain was measured using a numerical pain rating scale last week, ranging from 0 (no pain) to 10 (worst pain). ${ }^{33}$

\section{Sick Leave Register Data}

Sick leave was measured using data from the Norwegian Labor and Welfare Service, in which all individuals receiving any form of sick leave or disability benefits in Norway are registered. The data comprised all registered medical benefits individually traceable to each participant through social security numbers. Combining data on different types of medical benefits, we calculated the number of sick leave days during 12 months of follow-up after the interventions ended.

\section{Randomization and Blinding}

Potential participants were identified in the National Social Security System and invited through a letter. Invited participants completed a short eligibility questionnaire. Those who qualified were invited to an outpatient screening assessment. If they cleared this process, subjects were randomized to either the inpatient or outpatient program. A flexibly weighted randomization procedure was provided by the Unit of Applied Clinical Research (thirdparty) at the Norwegian University of Science and Technology (NTNU) to ensure that the rehabilitation center had enough participants to operate monthly groups during periods of low recruitment.

It was not possible to blind either the participants or the caregivers for treatment. Employees at the Norwegian Welfare and Labor Service, who were unaware of group allocation, provided sick leave data. The researchers were not blinded either. 


\section{Statistical Analysis}

A linear regression model was used to estimate the programs' effect on people with and without insomnia through the mean number of sick leave days during the 12month follow-up period. The precision of the estimates was assessed using 95\% confidence intervals. To test for interaction on an additive scale, we entered a product term (occupational program $\mathrm{x}$ insomnia) into the linear regression model. The analyses were adjusted for age, gender and education. We also conducted three sensitivity analyses to test the results' robustness. First, we repeated the main analyses, adjusting for diagnosis of sick leave and length of sick leave upon inclusion. Second, since an ISI score $\geq 15$ is often interpreted as moderate-to-severe insomnia, ${ }^{31}$ we repeated the main analysis using an ISI cutoff of 15 , rather than 11 , to dichotomize the participants into "no insomnia" and "clinically significant insomnia". The ISI cutoff of 15 was not chosen as our main analysis since this may produce several false positives. ${ }^{31}$ Third, we used a logistic regression model to estimate the probability of sustainable return to work (sustainable return to work was defined as one month without receiving medical benefits during follow-up). Fourth, since there is a considerable overlap, but unclear temporal effects, between insomnia and mental health conditions, ${ }^{22}$ we repeated the main analysis adjusting for depression.

All the analyses were performed using STATA 16 (StataCorp. 2019. Stata Statistical Software: Release 16. College Station, TX: StataCorp LP).

\section{Results}

Table 1 presents the baseline characteristics of the 163 participants stratified by occupational programs and insomnia. The proportion of participants who fulfilled the criteria for chronic insomnia at baseline was $56 \%(n=91)$. Overall, the mean total days with sick leave during the 12month follow-up were 114 (SD 86), and 49\% achieved sustainable return to work. Figure 1 shows the participant flow in the study.

Table 2 presents the mean differences in accumulated sick leave during 12 months of follow-up for each stratum of occupational program and insomnia, with a single reference category comprising people in a comprehensive inpatient program without insomnia. Compared with this reference category, people with insomnia had 12 (95\% $\mathrm{CI}-48$ to 24 ) fewer days with sick leave if they participated in the inpatient program and 46 (95\% CI 8-83) more days if they participated in the outpatient program. People

Table I Characteristics of the Study Population Stratified by Occupational Program and Insomnia

\begin{tabular}{|c|c|c|c|c|}
\hline & \multicolumn{4}{|c|}{ Occupational Rehabilitation Program } \\
\hline & \multicolumn{2}{|c|}{ Inpatient $(n=86)$} & \multicolumn{2}{|c|}{ Outpatient ( $n=77)$} \\
\hline & Insomnia ${ }^{a}$ & No Insomnia & Insomnia ${ }^{a}$ & No Insomnia \\
\hline Participants, \% (n) & $56(48)$ & $44(38)$ & $56(43)$ & $44(34)$ \\
\hline Age, mean (SD) & $46.2(9.1)$ & $46.3(8.3)$ & $46.9(10.2)$ & $43.0(10.3)$ \\
\hline Women \% (n) & $85(4 I)$ & $76(24)$ & $63(27)$ & $9 \mathrm{I}(3 \mathrm{I})$ \\
\hline Higher education \% $(n)^{b}$ & $33(16)$ & $42(16)$ & $40(17)$ & $50(17)$ \\
\hline \multicolumn{5}{|l|}{ Main diagnoses for sick-leave (ICPC-2) \% $(n)^{c}$} \\
\hline L- musculoskeletal & $58(28)$ & $68(26)$ & $49(21)$ & $53(18)$ \\
\hline P- psychological ${ }^{d}$ & $42(20)$ & $32(12)$ & $51(22)$ & $47(16)$ \\
\hline Number of days with sick leave at inclusion, median days (IQR) ${ }^{\mathrm{e}}$ & $200(165-263)$ & $211(161-273)$ & $202(172-287)$ & $219(190-244)$ \\
\hline Average pain $(0-10)$, mean $(S D)^{f}$ & $5.5(2.0)$ & $4.2(1.8)$ & $4.9(2.4)$ & $4.7(1.9)$ \\
\hline \multicolumn{5}{|l|}{ HADS, mean (SD) } \\
\hline Anxiety $(0-21)$ & $8.8(4.0)$ & $5.6(2.9)$ & $10.0(3.8)$ & $6.7(3.6)$ \\
\hline Depression $(0-2 \mathrm{I})$ & $6.6(4.6)$ & $4.6(3.3)$ & $7.9(3.7)$ & $4.5(3.6)$ \\
\hline Insomnia severity index (0-28), mean (SD) & $16.6(4.1)$ & $5.2(3.5)$ & $16.8(3.3)$ & $5.7(3.0)$ \\
\hline
\end{tabular}

Notes: ${ }^{a}$ Insomnia defined as an Insomnia Severity Index score $\geq 1 \mathrm{I}$. ${ }^{\mathrm{b}} \mathrm{College}$ or university. ${ }^{\mathrm{C} B a s e d}$ on data in the medical certificate from the National Social Security System Registry. ${ }^{\mathrm{d}}$ These include 13 participants with fatigue and one with perinatal distress. ${ }^{\mathrm{e}} \mathrm{Number}$ of days on sick leave during the last 12 months prior to inclusion. Measured as calendar days, not adjusted for graded sick-leave or part time job. ${ }^{\mathrm{f}}$ Average pain last week.

Abbreviation: HADS, Hospital Anxiety and Depression scale. 


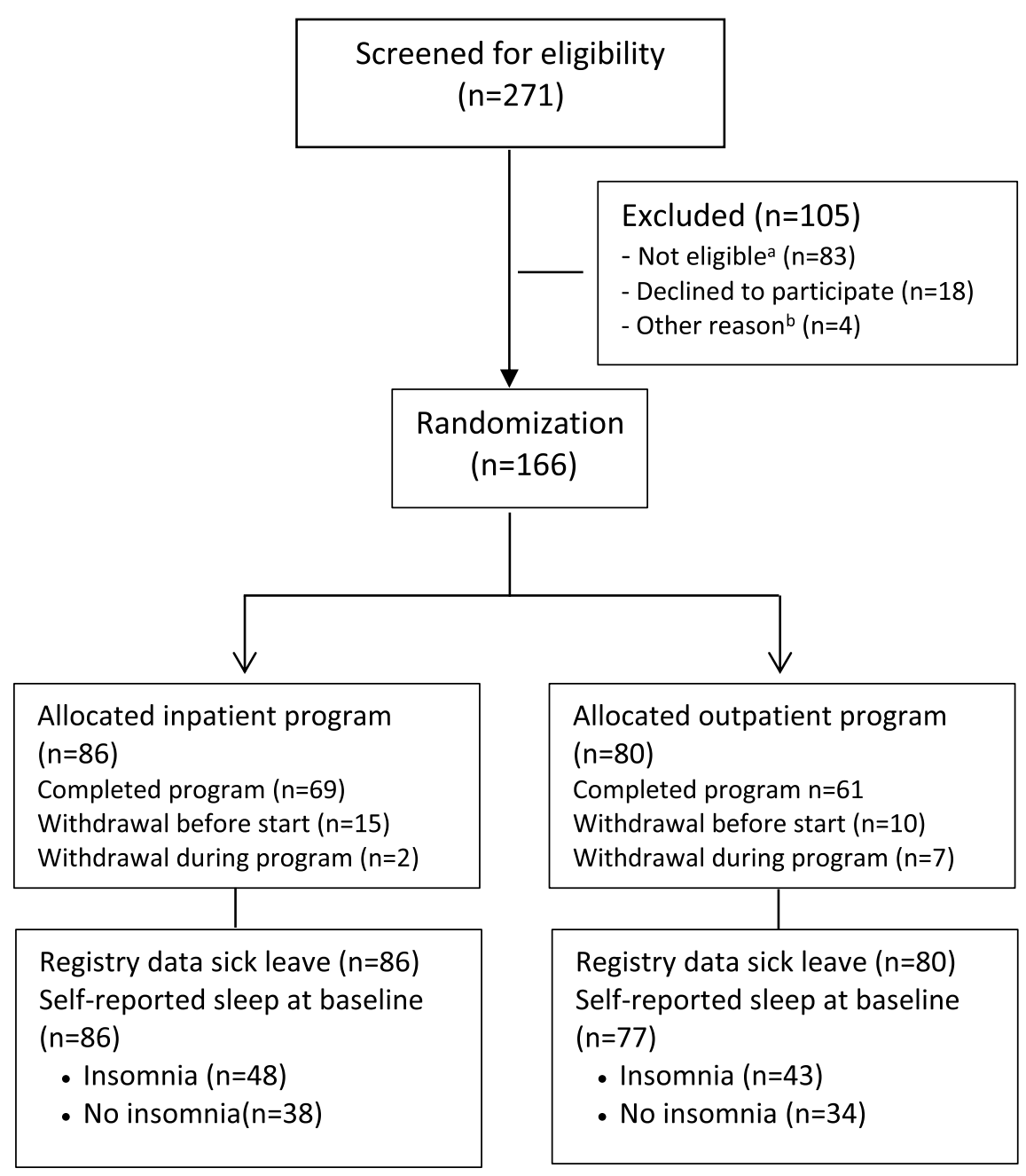

Figure I Flowchart of the participants in the study. ${ }^{a}$ Not eligible: Participating in another treatment program $(n=22)$, serious somatic/psychiatric illness $(n=I I)$, specialized treatment needs $(n=4)$, problems with functioning in groups $(n=3)$, surgery scheduled next 6 months $(n=2)$, insufficient language skills $(n=2)$, alcohol/drug abuse $(n=I)$, no longer on sick-leave $(n=10)$, medical assessment not completed $(n=15)$, not motivated $(n=6)$, inability to participate in an inpatient intervention $(n=7)$. ${ }^{\circ}$ Other reason: unknown $(n=4)$.

without insomnia in the outpatient program had the same number of days with sick leave (mean difference: $-3,95 \%$ $\mathrm{CI}-43$ to 37 ) as the reference category. We found statistical evidence of an interaction between intervention and insomnia $(\mathrm{p}=0.03)$. Figure 2 presents days of medical benefits throughout the 12 months of follow-up (cumulative mean) for individuals in each stratum of occupational program and insomnia.

\section{Sensitivity Analyses}

When adjusting for diagnosis of sick leave and length of sick leave at inclusion, the results remained largely unchanged. Similar associations were found when we used an ISI cutoff of $\geq 15$ or repeated the analyses, estimating the probability for sustainable return to work. Finally, adjusting for depression had only a negligible influence on our estimates (Supplementary material: Figure S1 and Tables S1-S3).

\section{Discussion}

In this subgroup analysis of a clinical trial on sick-listed individuals randomized to multimodal inpatient rehabilitation or a less-comprehensive outpatient program, the difference in cumulative sick leave during 12 months of follow-up was particularly evident among participants with insomnia. Individuals with insomnia in the multimodal inpatient rehabilitation program had substantially fewer days with sick leave than the outpatient group, but no difference between the programs was found in those without insomnia.

This is the first study to evaluate whether insomnia is associated with occupational rehabilitation's effectiveness 
Table 2 Associations of Occupational Program and Insomnia with Accumulated Sick Leave During 12 Months Follow-Up. Insomnia Measured by Insomnia Severity Index (ISI)

\begin{tabular}{|c|c|c|c|c|}
\hline & \multirow{2}{*}{$\begin{array}{l}\text { No. of } \\
\text { Persons }\end{array}$} & \multicolumn{3}{|c|}{ Days with Sick Leave } \\
\hline & & $\begin{array}{l}\text { Age Adjusted } \\
\text { Mean }\end{array}$ & $\begin{array}{l}\text { Age-Adjusted Mean Difference } \\
\qquad(95 \% \mathrm{Cl})^{\mathrm{a}}\end{array}$ & $\begin{array}{l}\text { Multi-Adjusted Mean Difference } \\
\qquad(95 \% \mathrm{Cl})^{\mathrm{ab}}\end{array}$ \\
\hline \multicolumn{5}{|l|}{ No insomnia ${ }^{c}$} \\
\hline Inpatient program & 38 & 105 & Reference & Reference \\
\hline Outpatient program & 34 & 105 & $0(-40$ to 39$)$ & $-3(-43$ to 37$)$ \\
\hline \multicolumn{5}{|l|}{ Insomnia $^{d}$} \\
\hline Inpatient program & 48 & 97 & $-8(-44$ to 28$)$ & $-12(-48$ to 24$)$ \\
\hline Outpatient program & 43 & 148 & 43 (5 to 80$)$ & 46 (8 to 83 ) \\
\hline
\end{tabular}

Notes: ${ }^{a}$ Mean difference: difference in number of sick leave days relative to the reference group of people in the inpatient program without insomnia. ${ }^{\mathrm{b}}$ Adjusted for age, sex and education. ' ISI-score <II. 'ISI-score $\geq 1$ I.

Abbreviations: $\mathrm{Cl}$, confidence interval; ISI, Insomnia Severity Index.

in future work participation in individuals with musculoskeletal and common mental health disorders. The results from our study complement evidence from observational and clinical studies indicating that insomnia is associated with sick leave and work disability. ${ }^{9-11}$ That our findings found a greater difference between the two programs in those with insomnia may be related to the multimodal inpatient rehabilitation program's comprehensive design. The inpatient rehabilitation incorporated principles that are known to be helpful for good sleep quality, such as physical exercise, ${ }^{25,26}$ psychoeducational sessions, including sleep guidance and fixed schedules. ${ }^{27}$ A consistent wakeup time is a recommended component in the treatment of insomnia, ${ }^{27}$ and regular physical activity has been suggested as an alternative treatment option for people with insomnia. ${ }^{34}$ In addition, the psychoeducational sessions

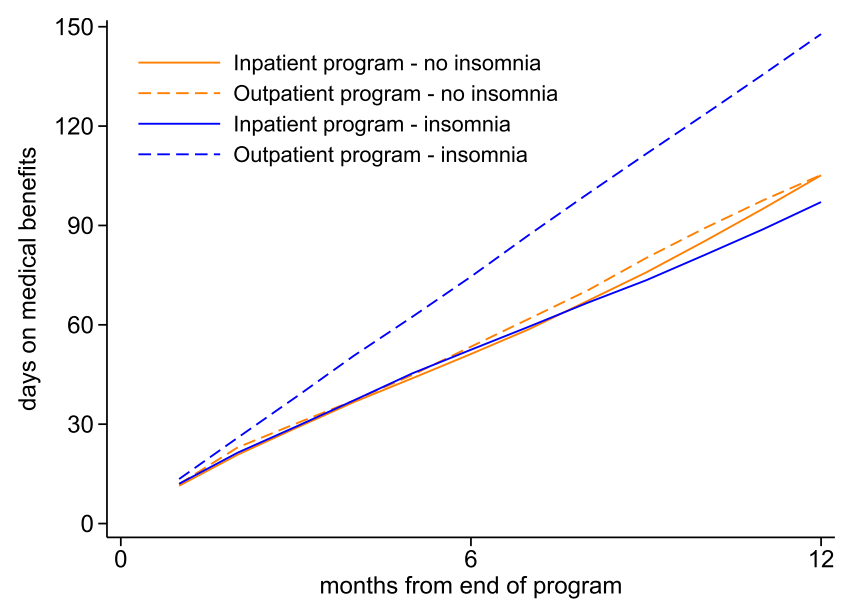

Figure 2 Sick leave days (cumulative mean) during 12 months of follow-up for participants in each stratum of occupational programme and insomnia. included sleep, management of stress and work life, and problem-solving techniques and coping strategies, which may exert positive effects on sleep quality. ${ }^{35,36}$ Furthermore, participants in the inpatient program broke away from regular life and had time to focus solely on their own situations. In contrast, participants in the outpatient program lived their regular lives at home. However, the outpatient program comprised regular acceptance and commitment therapy sessions that are also likely to improve sleep quality, ${ }^{37}$ but did not include sleep guidance or fixed schedules. Thus, the estimates in this study might be conservative.

The positive effect of the comprehensive inpatient program on future work participation among those with insomnia was considerable, compared with those with insomnia in the outpatient program. However, insomnia may be a proxy for health status, ie, people with comorbid chronic pain and insomnia have greater symptom severity, longer duration of symptoms, more disability and comorbidities, ${ }^{38}$ and the effect from inpatient rehabilitation may be related to a more complex mechanism than improvement of insomnia alone. For instance, although the data in the current study show that insomnia is prevalent among sick-listed workers with musculoskeletal pain or mental health conditions, it is well established that insomnia is bi-directionally related with pain and mental health conditions. ${ }^{16,21,22}$ Supplementary analyses with additional adjustment for depression had negligible influence on our estimates. However, the study design in the current study cannot verify the causal pathways between these conditions throughout the follow-up, that is, which factors that are likely to influence each other. In addition to improved 
sleep quality, therapies that target insomnia may improve pain, ${ }^{39,40}$ psychological well-being ${ }^{41,42}$ and fatigue. ${ }^{43}$ To understand this complexity, future studies should be designed to assess the effect of targeting insomnia in return to work interventions, and if and how specific principles from cognitive-behavioral therapy for insomnia should be combined with other dimensions of the multimodal inpatient program. Furthermore, more research is needed on which interventions are most effective for certain individuals. It has been suggested that subgroups should be identified based on prognostic return-to-work factors; ${ }^{7}$ therefore, our findings are important, as they suggest that insomnia should be addressed before sicklisted individuals take part in occupational rehabilitation programs, and that insomnia may assist in early identification of individuals at high risk for long-term sick leave.

This trial's strengths include the use of high-quality sick-leave registry data that provided complete and detailed data on sick leave and return to work throughout the long follow-up (12 months). Another strength is the detailed sleep questionnaire administered at the start of the intervention. Although primary researchers were not blinded in preparing for the data set, one of the authors was blinded to initiate and perform a separate analysis when conducting the primary outcome in the original study. ${ }^{24}$ Moreover, employees at the Norwegian Labor and Welfare Service who registered sick-leave data were unaware of the group allocation in this study. Another strength is that the Norwegian Labor and Welfare Service invited participants from among those who fit the eligibility criteria in the registry. However, only $38 \%$ (of 3808 invited) responded, and only 271 underwent a full clinical multidisciplinary eligibility assessment. Some limitations should be mentioned when interpreting the findings. First, we had no usual care control group and, therefore, no information on how these interventions compare with usual care. Although the inpatient program included a consistent wakeup time, information about sleep habits, and recommendations to improve sleep, the program did not include specific treatment components from the cognitive-behavioral therapy for insomnia. Unfortunately, we had no information about the use of sleep medication or if the participants had other sleep disorders (eg, obstructive sleep apnea, restless legs syndrome). It should also be noted that legislation, social security systems and occupational rehabilitation services differ between countries. Thus, generalizing our findings to other countries or settings with different services may not be straightforward.
Subgroup analysis itself presents some challenges as well. For instance, considering that our subgroup analysis was not part of the original study protocol, ${ }^{28}$ the participants were not randomized within subgroups by stratification. Although this might have created imbalances in possible confounders between the programs, it should be noted that insomnia at baseline was not influenced by the intervention itself or by prognostic characteristics that emerged after randomization. Finally, the study may have been underpowered to detect statistically significant interaction between insomnia and occupational programs on future work participation.

In conclusion, we found that the favorable effect of a multimodal inpatient program on future work participation was associated with insomnia. No difference between the programs was found in those without insomnia. These findings suggest that insomnia should be addressed specifically before individuals on sick leave are considered for participation in occupational rehabilitation, and that individuals with insomnia may benefit in particular from inpatient multimodal occupational rehabilitation.

\section{Data Sharing Statement}

The data are not available, as the participants have not consented to this.

\section{Ethical Approval}

The study was approved by the Regional Committee for Ethics in Medical Research (project no. 2012/1241 REK Mid-Norway). The study was conducted in accordance with the ethical standards of the 1964 Helsinki declaration and its later amendments. Informed consent was obtained from all individual participants included in the study.

\section{Clinical Trial Registration Number NCT01926574.}

\section{Acknowledgments}

We would like to thank all involved at Hysnes Rehabilitation Centre, Department of Pain and Complex Symptom Disorders and Department of Physical Medicine and Rehabilitation, at St. Olavs University Hospital, as well as the Norwegian Labour and Welfare Service (NAV) for their help with data collection and with conducting the study. We also want to thank project assistant Guri Helmersen for her valuable assistance. 


\section{Author Contributions}

All authors made a significant contribution to the work reported, whether that is in the conception, study design, execution, acquisition of data, analysis and interpretation, or in all these areas; took part in drafting, revising or critically reviewing the article; gave final approval of the version to be published; have agreed on the journal to which the article has been submitted; and agree to be accountable for all aspects of the work.

\section{Funding}

The Norwegian government (allocated through the Central Norway Regional Health Authority) and the Research Council of Norway funded this study. The work was also supported by a grant to Eivind Schjelderup Skarpsno from the Liaison Committee between the Central Norway Regional Health Authority (RHA) and the Norwegian University of Science and Technology (NTNU). The funders played no role in study design, data collection and analysis, writing up the results or deciding whether to publish this paper.

\section{Disclosure}

Dr Sigmund Østgård Gismervik reports grants from the Regional Health Authority of Central Norway, during the conduct of the study. Dr Lene Aasdahl reports grants from The Research Council of Norway, during the conduct of the study. The authors report no other potential conflicts of interest for this work.

\section{References}

1. Vos T, Flaxman AD, Naghavi M, et al. Years lived with disability (YLDs) for 1160 sequelae of 289 diseases and injuries 1990-2010: a systematic analysis for the Global Burden of Disease Study 2010. Lancet. 2012;380 (9859):2163-2196. doi:10.1016/S0140-6736(12)61729-2

2. Cullen KL, Irvin E, Collie A, et al. Effectiveness of workplace interventions in return-to-work for musculoskeletal, pain-related and mental health conditions: an update of the evidence and messages for practitioners. J Occup Rehabil. 2018;28(1):1-15. doi:10.1007/ s10926-016-9690-x

3. Lambeek LC, van Mechelen W, Knol DL, Loisel P, Anema JR. Randomised controlled trial of integrated care to reduce disability from chronic low back pain in working and private life. $B M J$. 2010;340(mar16 1):c1035. doi:10.1136/bmj.c1035

4. Anema JR, Steenstra IA, Bongers PM, et al. Multidisciplinary rehabilitation for subacute low back pain: graded activity or workplace intervention or both? A randomized controlled trial. Spine. 2007;32 (3):291-8; discussion 299-300. doi:10.1097/01.brs.0000253604. 90039.ad

5. Myhre K, Marchand GH, Leivseth G, et al. The effect of work-focused rehabilitation among patients with neck and back pain: a randomized controlled trial. Spine. 2014;39(24):1999-2006. doi:10.1097/ BRS.0000000000000610
6. Reme SE, Grasdal AL, Løvvik C, Lie SA, Øverland S. Work-focused cognitive-behavioural therapy and individual job support to increase work participation in common mental disorders: a randomised controlled multicentre trial. Occup Environ Med. 2015;72(10):745-752. doi:10.1136/oemed-2014-102700

7. Standal MI, Aasdahl L, Jensen C, et al. Subgroups of long-term sick-listed based on prognostic return to work factors across diagnoses: a cross-sectional latent class analysis. J Occup Rehabil. 2021;31(2):383-392. doi:10.1007/s10926-020-09928-5

8. Haldorsen EM, Grasdal AL, Skouen JS, Risa AE, Kronholm K, Ursin H. Is there a right treatment for a particular patient group? Comparison of ordinary treatment, light multidisciplinary treatment, and extensive multidisciplinary treatment for long-term sick-listed employees with musculoskeletal pain. Pain. 2002;95(1):49-63. doi:10.1016/S0304-3959(01)00374-8

9. Lallukka T, Kaikkonen R, Härkänen T, et al. Sleep and sickness absence: a Nationally Representative Register-Based Follow-Up Study. Sleep. 2014;37(9):1413-1425. doi:10.5665/sleep.3986

10. Jansson C, Alexanderson K, Kecklund G, Akerstedt T. Clinically diagnosed insomnia and risk of all-cause and diagnosis-specific disability pension: a nationwide cohort study. Sleep Disord. 2013;2013:209832. doi:10.1155/2013/209832

11. Canivet C, Staland-Nyman C, Lindeberg SI, Karasek R, Moghaddassi M, Ostergren PO. Insomnia symptoms, sleep duration, and disability pensions: a prospective study of Swedish workers. Int J Behav Med. 2014;21(2):319-328. doi:10.1007/s12529-0139315-0

12. Linton SJ, Bryngelsson IL. Insomnia and its relationship to work and health in a working-age population. J Occup Rehabil. 2000;10 (2):169-183. doi:10.1023/A:1009408204694

13. Alfoldi P, Wiklund T, Gerdle B. Comorbid insomnia in patients with chronic pain: a study based on the Swedish quality registry for pain rehabilitation (SQRP). Disabil Rehabil. 2014;36(20):1661-1669. doi:10.3109/09638288.2013.864712

14. Fang H, Tu S, Sheng J, Shao A. Depression in sleep disturbance: a review on a bidirectional relationship, mechanisms and treatment. J Cell Mol Med. 2019;23(4):2324-2332. doi:10.1111/ jcmm. 14170

15. Skarpsno ES, Mork PJ, Nilsen TIL, Steingrimsdottir OA, Zwart JA, Nilsen KB. The interplay between sleeplessness and high-sensitivity C-reactive protein on risk of chronic musculoskeletal pain. Longitudinal data from the Tromso Study. Sleep. 2019;42(9): zsz127. doi:10.1093/sleep/zsz127

16. Skarpsno ES, Mork PJ, Hagen K, Nilsen TIL, Marcuzzi A. Number of chronic nighttime insomnia symptoms and risk of chronic widespread pain and pain-related disability: the HUNT Study. Nat Sci Sleep. 2020;12:1227-1236. doi:10.2147/NSS.S284498

17. Skarpsno ES, Mork PJ, Nilsen TIL, Nordstoga AL. Influence of sleep problems and co-occurring musculoskeletal pain on long-term prognosis of chronic low back pain: the HUNT Study. J Epidemiol Community Health. 2020;74(3):283-289. doi:10.1136/jech-2019212734

18. Khurshid KA. Comorbid insomnia and psychiatric disorders: an update. Innov Clin Neurosci. 2018;15(3-4):28-32.

19. Neckelmann D, Mykletun A, Dahl AA. Chronic insomnia as a risk factor for developing anxiety and depression. Sleep. 2007;30 (7):873-880. doi:10.1093/sleep/30.7.873

20. Sivertsen B, Salo P, Mykletun A, et al. The bidirectional association between depression and insomnia: the HUNT study. Psychosom Med. 2012;74(7):758-765. doi:10.1097/PSY.0b013e3182648619

21. Skarpsno ES, Nilsen TI, Sand T, Hagen K, Mork PJ. Do physical activity and body mass index modify the association between chronic musculoskeletal pain and insomnia? Longitudinal data from the HUNT study, Norway. J Sleep Res. 2018;27(1):32-39. doi:10.1111/ jsr. 12580 
22. Alvaro PK, Roberts RM, Harris JK. A systematic review assessing bidirectionality between sleep disturbances, anxiety, and depression. Sleep. 2013;36(7):1059-1068. doi:10.5665/sleep.2810

23. Braathen TN, Veiersted KB, Heggenes J. Improved work ability and return to work following vocational multidisciplinary rehabilitation of subjects on long-term sick leave. J Rehabil Med. 2007;39 (6):493-499. doi:10.2340/16501977-0081

24. Gismervik S, Aasdahl L, Vasseljen O, et al. Inpatient multimodal occupational rehabilitation reduces sickness absence among individuals with musculoskeletal and common mental health disorders: a randomized clinical trial. Scand J Work Environ Health. 2020;46 (4):364-372. doi:10.5271/sjweh.3882

25. Kredlow MA, Capozzoli MC, Hearon BA, Calkins AW, Otto MW. The effects of physical activity on sleep: a meta-analytic review. J Behav Med. 2015;38(3):427-449. doi:10.1007/s10865-015-9617-6

26. Driver H, Taylor S. Exercise and sleep. Sleep Med Rev. 2000;4 (4):387-402. doi:10.1053/smrv.2000.0110

27. Riemann D, Baglioni C, Bassetti C, et al. European guideline for the diagnosis and treatment of insomnia. J Sleep Res. 2017;26 (6):675-700. doi:10.1111/jsr. 12594

28. Fimland MS, Vasseljen O, Gismervik S, et al. Occupational rehabilitation programs for musculoskeletal pain and common mental health disorders: study protocol of a randomized controlled trial. BMC Public Health. 2014;14:368. doi:10.1186/1471-2458-14-368

29. Hayes SC, Strosahl KD, Wilson KG. Acceptance and Commitment Therapy: An Experiential Approach to Behavior Change. New York: The Guilford Press; 2003.

30. Bastien $\mathrm{CH}$, Vallières $\mathrm{A}$, Morin $\mathrm{CM}$. Validation of the insomnia severity index as an outcome measure for insomnia research. Sleep Med. 2001;2(4):297-307. doi:10.1016/S1389-9457(00)00065-4

31. Morin CM, Belleville G, Bélanger L, Ivers H. The insomnia severity index: psychometric indicators to detect insomnia cases and evaluate treatment response. Sleep. 2011;34(5):601-608. doi:10.1093/sleep/ 34.5.601

32. Bjelland I, Dahl AA, Haug TT, Neckelmann D. The validity of the hospital anxiety and depression scale. An updated literature review. J Psychosom Res. 2002;52(2):69-77. doi:10.1016/S0022-3999(01) 00296-3
33. Cleeland CS, Ryan KM. Pain assessment: global use of the brief pain inventory. Ann Acad Med Singap. 1994;23(2):129-138.

34. Kline CE. The bidirectional relationship between exercise and sleep: implications for exercise adherence and sleep improvement. Am J Lifestyle Med. 2014;8(6):375-379. doi:10.1177/1559827614544437

35. Linton SJ. Does work stress predict insomnia? A prospective study. Br J Health Psychol. 2004;9(Pt 2):127-136. doi:10.1348/ 135910704773891005

36. Garefelt J, Platts LG, Hyde M, Magnusson Hanson LL, Westerlund H, Åkerstedt T. Reciprocal relations between work stress and insomnia symptoms: a prospective study. J Sleep Res. 2020;29 (2):e12949. doi:10.1111/jsr.12949

37. Salari N, Khazaie H, Hosseinian-Far A, et al. The effect of acceptance and commitment therapy on insomnia and sleep quality: a systematic review. BMC Neurol. 2020;20(1):300. doi:10.1186/ s12883-020-01883-1

38. Husak AJ, Bair MJ. Chronic pain and sleep disturbances: a pragmatic review of their relationships, comorbidities, and treatments. Pain Med. 2020;21(6):1142-1152. doi:10.1093/pm/pnz343

39. Vitiello MV, Rybarczyk B, Von Korff M, Stepanski EJ. Cognitive behavioral therapy for insomnia improves sleep and decreases pain in older adults with co-morbid insomnia and osteoarthritis. J Clin Sleep Med. 2009;5(4):355-362. doi:10.5664/jcsm.27547

40. Smith MT, Haythornthwaite JA. How do sleep disturbance and chronic pain inter-relate? Insights from the longitudinal and cognitive-behavioral clinical trials literature. Sleep Med Rev. 2004;8 (2):119-132. doi:10.1016/S1087-0792(03)00044-3

41. Cunningham JEA, Shapiro CM. Cognitive Behavioural Therapy for Insomnia (CBT-I) to treat depression: a systematic review. J Psychosom Res. 2018;106:1-12. doi:10.1016/j.jpsychores.2017.12.012

42. Carney CE, Edinger JD, Kuchibhatla M, et al. Cognitive behavioral insomnia therapy for those with insomnia and depression: a randomized controlled clinical trial. Sleep. 2017;40(4):4. doi:10.1093/sleep/zsx019

43. Taylor DJ, Peterson AL, Pruiksma KE, et al. Impact of cognitive behavioral therapy for insomnia disorder on sleep and comorbid symptoms in military personnel: a randomized clinical trial. Sleep. 2018;41(6):6. doi:10.1093/sleep/zsy069
Nature and Science of Sleep

\section{Publish your work in this journal}

Nature and Science of Sleep is an international, peer-reviewed, open access journal covering all aspects of sleep science and sleep medicine, including the neurophysiology and functions of sleep, the genetics of sleep, sleep and society, biological rhythms, dreaming, sleep disorders and therapy, and strategies to optimize healthy sleep.
The manuscript management system is completely online and includes a very quick and fair peer-review system, which is all easy to use. Visit http://www.dovepress.com/testimonials.php to read real quotes from published authors. 